\title{
TECHNOLOGY OF PRODUCING THE CONTACT CONNECTIONS OF SUPERCONDUCTOR METAL-SHEATHED CABLE
}

\section{ТЕХНОЛОГИЯ ИЗГОТОВЛЕНИЯ КОНТАКТНЫХ СОЕДИНЕНИЙ СВЕРХПРОВОДЯЩЕГО КАБЕЛЯ В МЕТАЛЛИЧЕСКОЙ ОБОЛОЧКЕ}

\author{
Andrzej JAKUBOWSKI \\ Maritime University of Szczecin
}

\begin{abstract}
:
The technology of producing the current contact connections on the superconductor cable edges is presented. This lead cable is used as one of the major elements of the magnetic system in thermonuclear reactor construction, actuality for modern world energy. The technology is realized by the radial draft of metal thin-walled tube on the conductor's package. The filling of various profiles by round section wire is optimized. Geometrical characteristics of the dangerous crosssection (as a broken ring) of thin-walled tube injured by the sector cut-out are accounted. The comparative strength calculation of the solid and injured tubes at a longitudinal compression and lateral bending is acted. The radial draft mechanism of cylindrical thin-walled sheath with the wire packing is designed. The necessity to use the nonlinear theory for the sheaths calculate is set. The resilient co-operation of wires as the parallel located cylinders with the contact stripes of rectangular form is considered.
\end{abstract}

\section{Резюме:}

Предложена технология изготовления токоподводящих контактных соединений по краям сверхпроводящего кабеля как одного из важнейших элементов магнитной системы в конструкции термоядерного реактора, актуального для современной мировой энергетики. Технология реализуется за счет радиального обжатия тонкостенной металлической трубки на пакет проводников. Оптимизировано заполнение различных профилей проволокой круглого сечения. Рассчитаны геометрические характеристики опасного поперечного сечения (разомкнутого кольца) тонкостенной трубы, ослабленной секторальным вырезом. Проведен сравнительный расчет прочности цельной и ослабленной трубы при продольном сжатии и поперечном изгибе. Смоделирован механизм радиального обжатия тонкостенной цилиндрической оболочки с проволочной паковкой и установлена необходимость использования нелинейной теории расчета оболочки. Рассмотрено упругое взаимодействие проволок как параллельно расположенных цилиндров, между которыми образуются контактные площадки в форме прямоугольной полосы.

Key words: superconductor cable, contact connection, wire, packing, thin-walled tube, radial draft, contact

Ключевые слова: сверхпроводящий кабель, контактное соединение, проволока, паковка, тонкостенная труба, радиальное обжатие, контакт

\section{ВВЕДЕНИЕ}

Мировое сообщество индустриально развитых стран обеспокоено проблемой обеспеченности энергоносителями. По разным данным разведанных запасов нефти хватит примерно на 20-30 лет, природного газа на 100-150 лет, а каменного угля на 300 лет. Запасы урановой руды тоже очень ограничены. По данным ООН потребности мира в электроэнергии к середине XXI века увеличатся в 4-6 раз. В связи с этим США, Россия, Китай, Индия, Япония, Южная Корея и Европейский Союз подписали соглашение о создании Международной организации по строительству первого Международного термоядерного экспериментального реактора (ИТЭР), энергетическая мощность которого должна составить 500 МВт. Разработка технического проекта ИТЭР была закончена в 2001 году. Реактор было намечено построить в городе Кадараш на юге Франции. Его возведение планировалось начать в 2009 году, и завершить строительство в 2016 году. При положительных результатах испытаний экспериментального реактора работы предполагается продолжить, а внедрение в реальную энергетику реализовать примерно в середине XXI века. При этом после экспериментального реактора намечено строительство так называемого коммерческого прототипа. Так, по предположению ученых к 2050 году начнут работать промышленные энергетические станции с термоядерными реакторами [1].

Планируется запустить процесс термоядерного синтеза, аналогичного тому, который происходит на 
Солнце. В случае успеха будет открыт способ получения экологически чистой энергии В неограниченном количестве. При термоядерном синтезе энергия высвобождается при взаимодействии атомных ядер изотопов водорода дейтерия $и$ трития, которые выступают в качестве топлива. Такого топлива требуется незначительное количество, но при этом синтезе высвобождается в 10 миллионов раз больше энергии, чем при сжигании ископаемого топлива. При слиянии дейтерия и трития происходит образование лития с выделением энергии в двадцать раз большей, чем при делении атомов урана. Запасы дейтерия на Земле велики. В одном кубометре морской воды находится около 33 г дейтерия. В стакане воды можно получить из дейтерия тепло, сопоставимое с теплом, выделяемым при сгорании 200 л бензина. Дейтерий можно получить с помощью недорогих технологий электролиза воды. В природе трития нет. Он вырабатывается при эксплуатации атомных тяжеловодных реакторов из изотопов лития и считается отходом производства. Если удастся создать рабочий термоядерный реактор, то он будет потреблять очень небольшое количество лития и дейтерия. Реактор с электрической мощностью 1 ГВт будет сжигать около 100 кг дейтерия и 300 кг лития в год. При таком потреблении содержащегося в воде дейтерия хватит человечеству на многие миллионы лет для снабжения энергией, а запасов лития минимум на 70 тысяч лет. По мнению ученых один грамм термоядерного топлива способен заменить 11 тонн каменного угля. Кроме того, термоядерная энергия видимо будет более чистой по сравнению с топливной энергетикой и более безопасной по сравнению с ядерной [1].

Важнейшим элементом реактора является сверхпроводящий кабель, по краям которого необходимо создать контактные соединения (KC) для подвода тока. Разработана технология изготовления КС тороидальной обмотки ИТЭР, реализуемая радиальным обжатием (рис. 1) медной трубки на пакет проводников (рис. 2) [1].

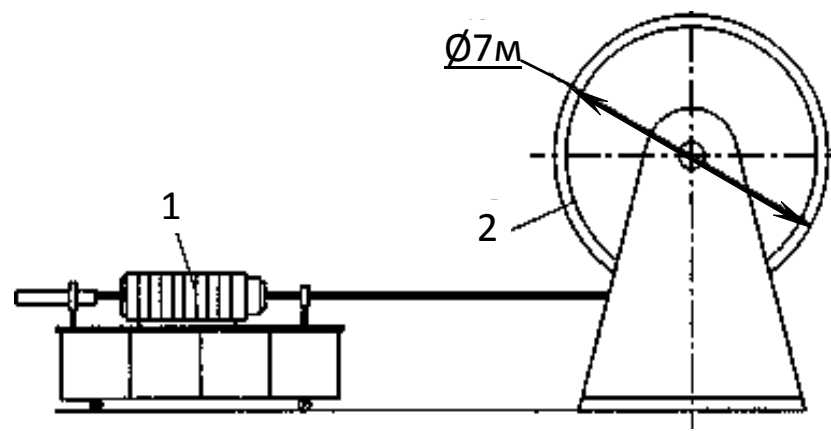

Рис. 1 Принципиальная схема обжатия КС сверхпроводников: 1 - установка для радиального обжатия;

2 - катушка кабеля сверхпроводника

Проведенные в 2001 г. в Японии испытания показали высокие технические характеристики КС, в частности, по электрическому сопротивлению контактной зоны соединений. Следует отметить, что технология и оборудование может быть использована для получения КС широкого целевого назначения [1].
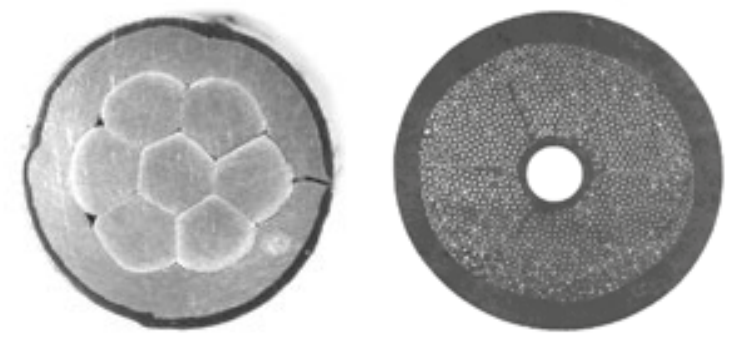

Puс. 2 Поперечные сечения КС после обжатия

\section{ПТИМИЗАЦИЯ ПАКОВКИ МНОГОЖИЛЬНЫХ КАБЕЛЕЙ}

Многие, главным образом электротехнические, устройства и коммуникации предполагают использование многожильных кабелей, представляющих собой паковку проволок различного сечения в полимерной, резиновой, металлической оболочке с изоляцией или без нее (рис. 3).

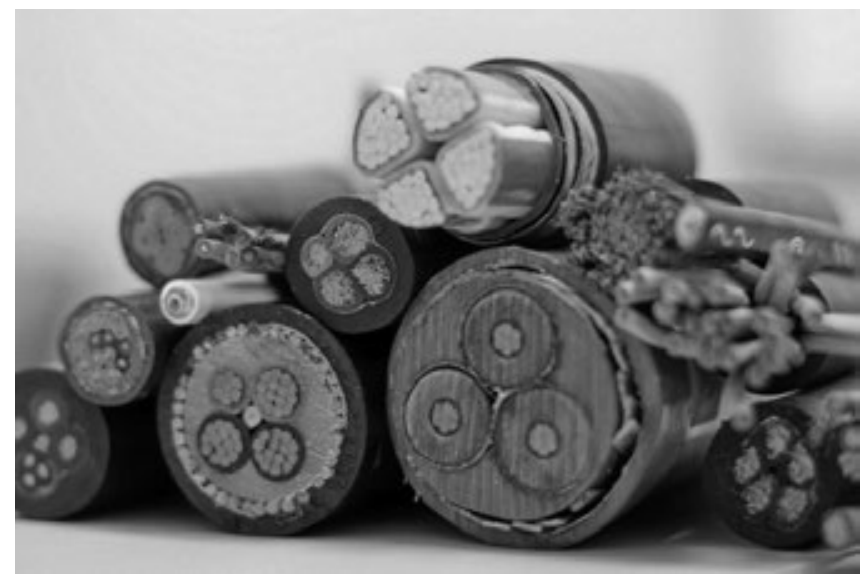

Рис. 3 Примеры различного исполнения кабелей

Например, гибкие шланговые кабели высокого напряжения используют для подачи электроэнергии к землеройным и горнодобывающим машинам или в лифтовых установках; бронированные кабели используют в магистральных линиях дальней связи, в силовых и осветительных установках, в линиях электропередачи высокого напряжения; маслонаполненные кабели высокого давления в стальном трубопроводе нашли применение на электростанциях при соединении повышающих трансформаторов, в районах с интенсивной застройкой, а также при необходимости прокладки через водные преграды.

Ведущиеся разработки оборудования магнитных систем термоядерного реактора касаются, в том числе, сверхпроводящего кабеля высокого напряжения [1]. В качестве такого изделия может быть использована паковка медной проволоки круглого сечения в тонкостенной металлической (медной или стальной) оболочке (трубке). В зависимости от целевого назначения кабеля укладка проволоки (жил) в нем может быть произвольная или упорядоченная, а оболочка круглого, шестигранного, прямоугольного или любого другого поперечного сечения (рис. 4). 


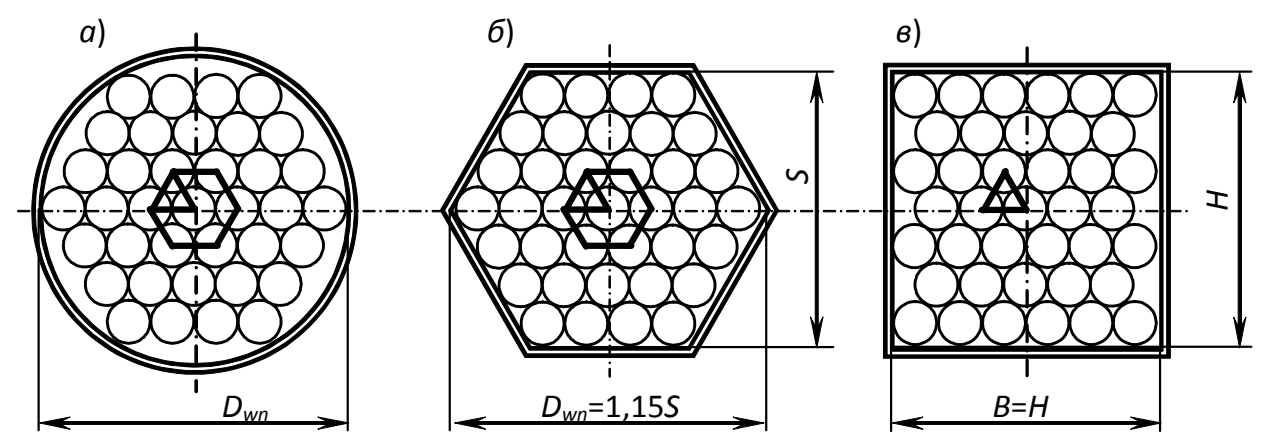

Рис. 4 Сечения паковок различного исполнения с упорядоченным расположением проволок в трубке круглого (a), шестигранного (б), прямоугольного (в) профиля

Важным критерием выбора того или иного исполнения является плотность заполнения оболочки проволокой. Этот показатель удобно выражать коэффициентом заполнения [2]:

$$
K=\frac{\sum_{i=1}^{n} A_{i}}{A_{w n}}=\frac{A_{d} \cdot n}{A_{w n}}
$$

где:

$\sum A_{i}-$ площадь, занимаемая паковкой,

$\mathrm{A}_{\mathrm{d}}=-\pi \mathrm{d}^{2} / 4$ площадь поперечного сечения проволоки диаметром d,

$\mathrm{N}$ - количество проволок в паковке,

$\mathrm{A}_{\mathrm{wn}}$ - площадь сечения полости трубки.

Стремление достичь более плотной паковки объясняется рациональным использованием материала и уменьшением объема воздушных каналов. Это реализуется упорядоченной укладкой проволоки и выражается коэффициентом заполнения $\mathrm{K} \leq 1$. Очевидно, что при произвольной, беспорядочной укладке образуются большие просветы, и паковка становится наименее плотная.

При упорядоченном расположении проволок одинакового диаметра в круглой оболочке в сечении формируются треугольные ячейки и образуются ортогональные слои в виде правильного шестигранника (см. рис. 4, a). Диаметр $D_{w n}$ полости оболочки зависит от диаметра d используемой проволоки и необходимого количества ее слоев $\mathrm{m}$.

Однако, при упорядоченной укладке в круглую оболочку остаются значительные свободные зоны, куда могут "сваливаться" проволочки крайнего слоя, поэтому существует большая вероятность нарушения регулярности укладки. В этом отношении при том же наборе проволок целесообразно использовать шестигранную оболочку (см. рис. 4, б), исключающую такую вероятность.

При укладке проволоки в прямоугольную оболочку упорядоченное и наиболее устойчивое расположение проволочек также сопровождается формированием треугольных ячеек (см. рис. 4, в), но слои следует рассматривать отдельно - горизонтальные $\mathrm{m}_{1}$ и вертикальные $\mathrm{m}_{2}$. Они определяют соответственно ширину В и высоту Н полости оболочки.

При упорядоченной и устойчивой укладке проволоки в квадратную оболочку, когда В=H, появляется жесткая взаимосвязь между слоями в обоих направлениях:

$$
m_{1}=m=\sqrt{3} \cdot\left(m_{2}-1\right) / 2+1
$$

В случае неустойчивой упорядоченной укладки проволоки В прямоугольную оболочку, когда образуются ячейки четырехугольной формы, коэффициент заполнения не зависит от количества слоев ни в одном из направлений и является величиной постоянной.

Таким образом, наиболее компактная паковка формируется при упорядоченной укладке проволоки с использованием шестигранной оболочки. На рис. 5 изображен график изменения коэффициента заполнения в зависимости от количества слоев проволоки для некоторых исполнений паковки. Результаты вычисления коэффициента заполнения представлены в табл. 1 [2].

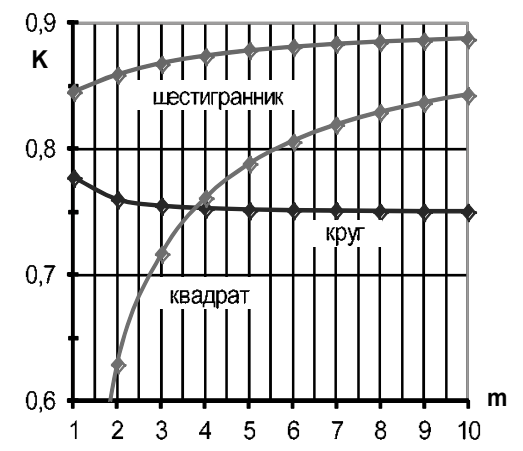

Рис. 5 Коэффициент заполнения

\section{ГЕОМЕТРИЯ ОСЛАБЛЕННОГО СЕЧЕНИЯ ТРУБЫ}

Соединения трубных элементов выполняют различными способами, в том числе, посредством сварки. В этом случае часто прибегают к вырезанию в них «окошек», что приводит к фактическому ослаблению труб. Поэтому при проектировании сварных конструкций для обеспечения их безотказной работы проводят расчет конструктивных элементов на прочность, а это непосредственно связано с определением геометрических характеристик поперечных сечений [3].

Принимая во внимание, что в качестве трубных элементов конструкций наиболее часто используют трубы круглого профиля, рассмотрим поперечное сечение в форме кольца со срединным диаметром $D=2 \mathrm{R}$ и толщиной стенки t (рис. 6). 
Табл. 1

Сводная таблица расчета коэффициента заполнения паковки различного профиля

\begin{tabular}{cccc}
\hline Паковка & $\begin{array}{c}\text { Площадь поперечного сечения } \\
\text { полости трубки, } \mathbf{A}_{\text {wn }}\end{array}$ & $\begin{array}{c}\text { Количество проволок } \\
\text { в паковке, } \mathbf{~}\end{array}$ & $\begin{array}{c}\text { Коэффициент заполнения } \\
\text { паковки, } \mathbf{K}\end{array}$ \\
\hline & круг & \\
4 & $\frac{\pi D_{w n}^{2}}{4}=\frac{\pi d^{2}}{4} \cdot(2 m+1)^{2}$ & $3 m(m+1)+1$ & $\frac{3 m(m+1)+1}{4 m(m+1)+1}$
\end{tabular}

шестигранник

$$
\frac{3 S D_{w n}}{4}=\frac{\sqrt{3} S^{2}}{2}=\frac{\sqrt{3} d^{2}}{2} \cdot(\sqrt{3} m+1)^{2}
$$$$
3 m(m+1)+1
$$$$
0,9 \cdot \frac{3 m(m+1)+1}{3 m(m+1,15)+1}
$$

прямоугольник

$$
B H=d^{2} m_{1} \cdot\left[\frac{\sqrt{3}}{2} \cdot\left(m_{2}-1\right)+1\right]
$$

$$
\left(m_{1}-\frac{1}{2}\right) \cdot m_{2}
$$

$$
0,9 \cdot \frac{1-1 / 2 m_{1}}{1+2 / 13 m_{2}}
$$

квадрат

$$
B^{2}=d^{2} m^{2}
$$

$$
\frac{2}{\sqrt{3}} \cdot\left(m-\frac{1}{2}\right) \cdot\left(m-\frac{2}{15}\right)
$$

$0,9 \cdot\left(1-\frac{1}{2 m}\right) \cdot\left(1-\frac{2}{15 m}\right)$

прямоугольник

$$
B H=d^{2} m_{1} m_{2}
$$

$m_{1} m_{2}$

Тогда в случае ослабления трубы в расчетах следует рассматривать опасное сечение в зоне ослаблениятонкостенный контур незамкнутого кольца со срединным радиусом R=D/2, толщиной $t$ и углом разделки $\beta=2 \pi-\alpha$ (см. рис. 6).

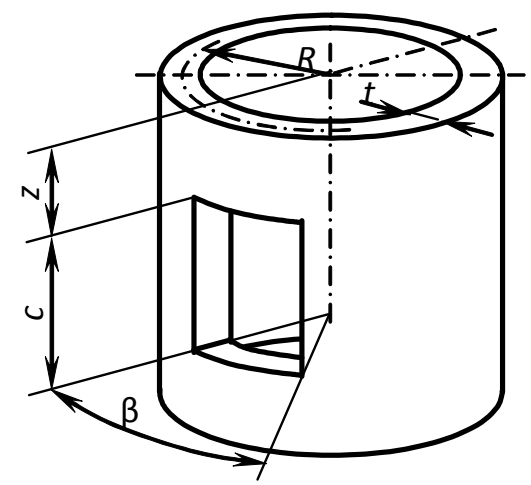

Рис. 6 Схема ослабленной трубы

Выделим из сечения элементарный участок площадью $d A=t \cdot R \cdot d \phi$ (рис. 7). Тогда площадь незамкнутого кольца равна [3]:

$$
A=\int_{A} d A=R \cdot t \cdot \alpha
$$

Чтобы определить другие геометрические характеристики сечения, необходимо установить положение его центра тяжести (точки С на рис. 7). С этой целью введем систему вспомогательных координатных осей $\mathrm{x}_{0}$ и $\mathrm{y}_{0}$ с началом отсчета в точке 0 , являющейся центом срединной линии сечения (центром окружности). Очевидно, что точка С будет лежать на оси $\mathrm{Y}_{0}$, т.е. горизонтальная координата центра тяжести сечения равна нулю $\left(\mathrm{x}_{\mathrm{C}}=0\right)$, а вертикальную координату Ус нужно найти.

Статический момент сечения относительно оси $\mathrm{x}_{0}$ равен [3]:

$$
S_{x_{0}}=\int_{A} y \cdot d A=2 \cdot R^{2} \cdot t \cdot \sin \frac{\alpha}{2}
$$

где:

$\mathrm{y}=\mathrm{R} \cdot \cos \phi$ - вертикальная координата выделенного элементарного участка.

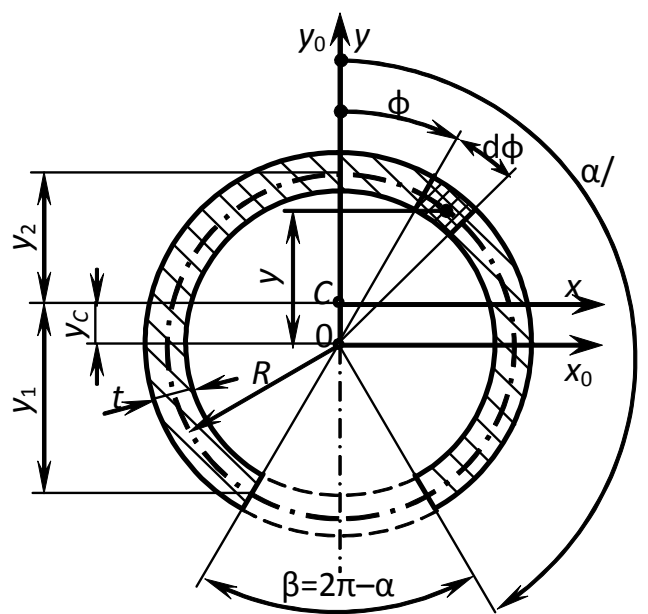

Рис. 7 Расчетная схема поперечного сечения ослабленной трубы 
Тогда с учетом выражения (3) находим [3]:

$$
y_{C}=\frac{S_{x_{0}}}{A}=\frac{2 \cdot R}{\alpha} \cdot \sin \frac{\alpha}{2}
$$

Осевой момент инерции сечения относительно оси $\mathrm{x}_{0}$ равен [3]:

$$
I_{x_{0}}=\int_{A} y^{2} \cdot d A=\frac{R^{3} \cdot t}{2} \cdot(\alpha+\sin \alpha)
$$

Тогда с учетом выражений (3), (5) и (6) осевой момент инерции относительно центральной оси х равен [3]:

$$
I_{x}=I_{x_{0}}-y_{C}^{2} \cdot A=\frac{\alpha \cdot R^{3} \cdot t}{2} \cdot\left(1+a-2 \cdot b^{2}\right)
$$

Здесь приняли:

$$
a=\frac{\sin \alpha}{\alpha}, \quad b=\frac{2}{\alpha} \cdot \sin \frac{\alpha}{2}
$$

Осевой момент инерции сечения относительно другой центральной оси у с учетом координаты $\mathrm{x}=\mathrm{R} \cdot \sin \phi$ равен [3]:

$$
I_{y}=I_{y_{0}}=\int_{A} x^{2} \cdot d A=\frac{\alpha \cdot R^{3} \cdot t}{2} \cdot(1-a)
$$

Радиусы инерции сечения определяются с учетом выражений (3), (7) и (9) следующим образом [3]:

$$
\begin{aligned}
& i_{x}=\sqrt{\frac{I_{x}}{A}}=R \cdot \sqrt{\frac{1+a-2 \cdot b^{2}}{2}} \\
& i_{y}=\sqrt{\frac{I_{y}}{A}}=R \cdot \sqrt{\frac{1-a}{2}} .
\end{aligned}
$$

Моменты сопротивления сечения вычисляют с учетом координат точек на срединной линии тонкостенного контура, наиболее удаленных от центральных осей х и у (см. рис. 7): где:

$$
\begin{aligned}
& \left|y_{1}\right|=\left|-R \cdot \cos \frac{\beta}{2}-y_{C}\right|=R \cdot\left(b-\frac{a}{b}\right) ; \\
& y_{2}=R-y_{C}=R \cdot(1-b) ; \quad x_{\max }=R,
\end{aligned}
$$

$\mathrm{y}_{\max }=|\mathrm{y} 1| \geq \mathrm{y} 2$

Тогда моменты сопротивления с учетом выражений (7), (9) и (11) равны [3]:

$$
\begin{aligned}
& W_{x}=\frac{I_{x}}{y_{\max }}=\frac{\alpha \cdot R^{2} \cdot t}{2} \cdot \frac{1+a-2 \cdot b^{2}}{b-a / b} ; \\
& W_{y}=\frac{I_{y}}{x_{\max }}=\frac{\alpha \cdot R^{2} \cdot t}{2} \cdot(1-a) .
\end{aligned}
$$

Выведенные выражения в полной мере описывают геометрические характеристики опасного сечения ослабленной круглой тонкостенной трубы. Эти формулы можно представить (табл. 2), объединив входящие в них тригонометрические функции а и b в коэффициенты:

$$
\begin{aligned}
& k_{1}=1+a-2 \cdot b^{2} ; \quad k_{2}=1-a \\
& k_{3}=b-a / b . \quad k_{4}=1-b .
\end{aligned}
$$

Графическое изображение зависимостей введенных коэффициентов и тригонометрических функций от целостности контура сечения трубы наглядно отображает закономерность их изменения (рис. 8). Из диаграммы следует, что увеличение угла разделки трубы сопровождается стремительным уменьшением всех геометрических характеристик ослабленного

\begin{tabular}{|c|c|c|c|}
\hline Геометрические характеристики сечения & трубы о & слабленной & $\begin{array}{c}\text { трубы цельной, } \\
\text { когда } \alpha=2 \pi, a=0, b=0 \text {, } \\
k_{1}=k_{2}=k_{3}=k_{4}=1\end{array}$ \\
\hline Площадь & $A=R$ & $\cdot t \cdot \alpha$ & $A_{0}=2 \pi \cdot R \cdot t$ \\
\hline $\begin{array}{l}\text { Координаты центра тяжести в системе } \\
\text { вспомогательных осей }\end{array}$ & $x_{C}=0$ & $y_{C}=R \cdot b$ & $x_{C}=0 \quad y_{C}=0$ \\
\hline Осевые моменты & $I_{x}=\frac{\alpha \cdot R^{3} \cdot t}{2} \cdot k_{1}$ & $I_{y}=\frac{\alpha \cdot R^{3} \cdot t}{2} \cdot k_{2}$ & $I_{x_{0}}=I_{y_{0}}=\pi \cdot R^{3} \cdot t$ \\
\hline Радиусы инерции & $i_{x}=R \cdot \sqrt{\frac{k_{1}}{2}}$ & $i_{y}=R \cdot \sqrt{\frac{k_{2}}{2}}$ & $i_{x_{0}}=i_{y_{0}}=R \cdot \frac{1}{\sqrt{2}}$ \\
\hline $\begin{array}{l}\text { Координаты точек, наиболее удаленных } \\
\text { от центральных осей }\end{array}$ & $x_{\max }=R$ & $y_{\text {max }}=\left|y_{1}\right|=R \cdot k_{3}$ & $x_{\max }=y_{\max }=R$ \\
\hline Моменты сопротивления & $W_{x}=\frac{\alpha \cdot R^{2} \cdot t}{2} \cdot \frac{k_{1}}{k_{3}}$ & $W_{y}=\frac{\alpha \cdot R^{2} \cdot t}{2} \cdot k_{2}$ & $W_{x_{0}}=W_{y_{0}}=\pi \cdot R^{2} \cdot t$ \\
\hline
\end{tabular}
сечения. Также видно, что геометрические характеристики ослабленного сечения относительно оси, параллельной разделке, всегда меньше характеристик относительно оси, перпендикулярной разделке. Кроме того, при $\beta \rightarrow 0$, когда $\mathrm{k}_{1}=\mathrm{k}_{2}=\mathrm{k}_{3}=\mathrm{k}_{4} \rightarrow 1$, они приближаются к характеристикам кольца. Достоверность приведенных выкладок подтверждается их полным соответствием известным формулам для замкнутого круглого тонкостенного сечения, когда $\beta=0$ (см. табл. 2) [4].

Табл. 2

Сводная таблица расчета коэффициента заполнения паковки различного профиля 


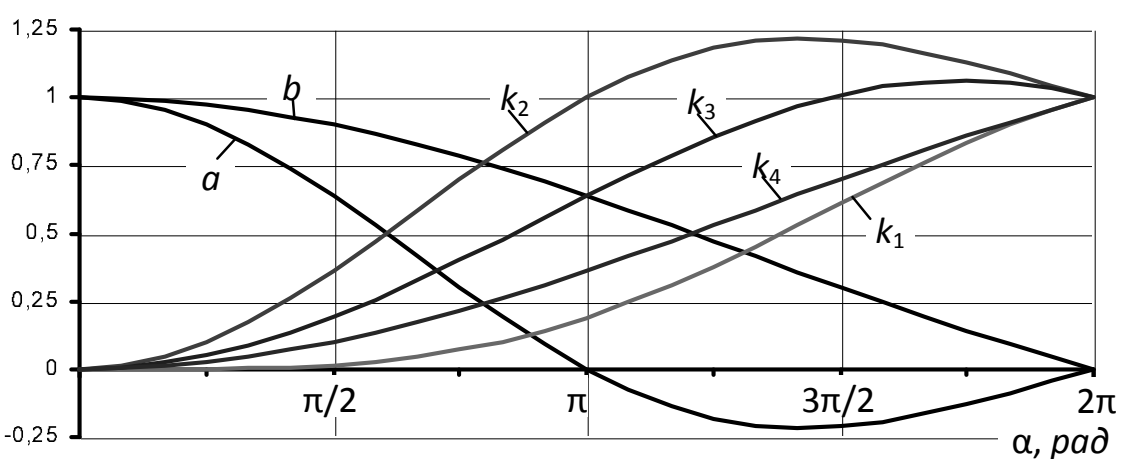

Рис. 8 Зависимости коэффициентов $k_{1}, k_{2}, k_{3}, k_{4}$ и функций а и $b$ от угла $\alpha$

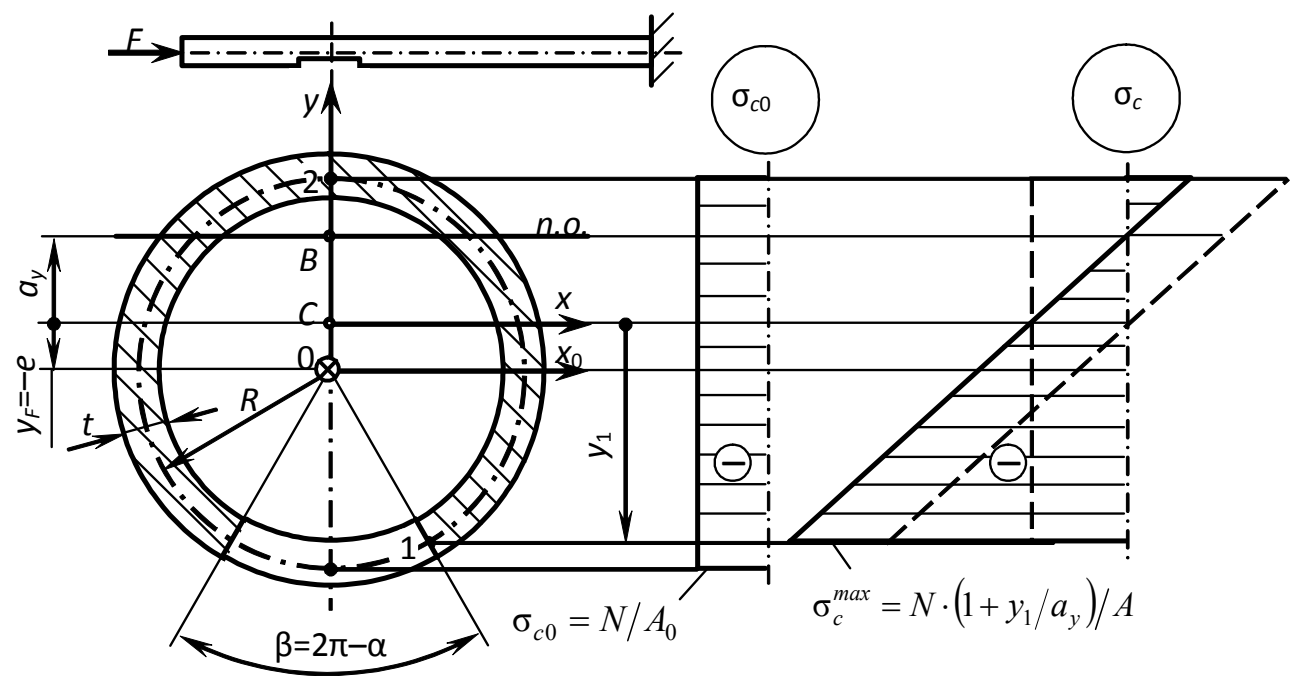

Рис. 9 Расчетная схема сжатия ослабленной трубы

\section{СРАВНЕНИЕ ПРОЧНОСТИ ЦЕЛЬНОЙ И ОСЛАБЛЕННОЙ ТРУБЫ}

Рассмотрим две типовые схемы нагружения отдельно взятой прямой трубы круглого сечения схему продольного сжатия и схему поперечного изгиба сосредоточенной силой F. Установим, во сколько раз уменьшится прочность в случае необходимости ее ослабления [5].

Схема продольного нагружения (рис. 9). В этом случае сосредоточенная сжимающая сила $\mathrm{F}$ действует вдоль продольной оси прямой цельной трубы, испытывающей осевое сжатие. Напряжения во всех ее сечениях равны [3]:

$$
\sigma_{c 0}=\frac{N}{A_{0}}=\frac{-F}{2 \pi \cdot R \cdot t}
$$

где:

$\mathrm{N}=-\mathrm{F}-$ нутренняя продольная сила, возникающая в поперечных сечениях сжатой трубы (знак «-» свидетельствует о деформации сжатия).

Ослабленная труба, находящаяся под действием той же силы F, в зоне ослабления испытывает внецентренное сжатие. Это вызвано тем, что центр тяжести разомкнутого кольца (точка С на рис. 9) смещен относительно геометрической оси трубы (точки 0) на величину $\mathrm{e}=\mathrm{R} \cdot \mathrm{b}$.

Следовательно, координата точки приложения силы в системе центральных осей незамкнутого кольца $\mathrm{yF}=-\mathrm{e}$.
Тогда напряжения в наиболее опасной точке ослабленного сечения равны [3]:

$$
\sigma_{c}=\frac{N}{A} \cdot\left(1+\frac{y_{F} \cdot y_{1}}{i_{x}^{2}}\right)=\frac{N}{A} \cdot\left(1-\frac{y_{1}}{a_{y}}\right)
$$

Здесь координата наиболее опасной точки сечения (точки 1) в системе центральных осей незамкнутого кольца $\mathrm{y}_{1}=-\mathrm{R} \cdot \mathrm{k}_{3}$, а положение нейтральной оси сечения определяется длиной отрезка ВС [3]:

$$
a_{y}=-\frac{i_{x}^{2}}{y_{F}}=\frac{i_{x}^{2}}{e}=\frac{R^{2} \cdot k_{1} / 2}{R \cdot b}=R \cdot \frac{k_{1}}{2 \cdot b}
$$

Тогда формула (15) принимает вид:

$$
\sigma_{c}^{\max }=\frac{-F}{\alpha \cdot R \cdot t} \cdot \frac{k_{2}}{k_{1}}
$$

где:

$k_{1}+2 b \cdot k_{3}=1-a=k_{2}$.

Отношение выражений (14) и (17) позволяет установить степень уменьшения прочности сжатой трубы при ее ослаблении, зависящую исключительно от геометрии выреза:

$$
\frac{\sigma_{c}^{\max }}{\sigma_{c 0}}=\frac{1}{1-\frac{\beta}{2 \pi}} \cdot \frac{k_{2}}{k_{1}}
$$


Схема поперечного нагружения (рис. 10).

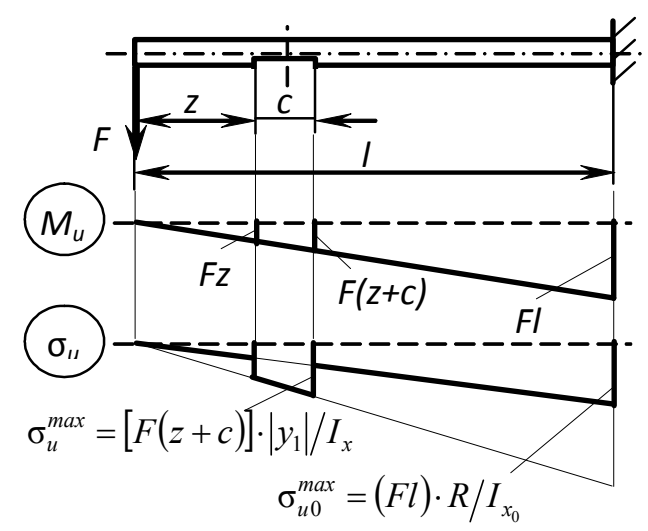

Рис. 10 Расчетная схема изгиба ослабленной трубы

В наиболее неблагоприятном случае, когда труба представляет собой консоль длиной I, нагруженную на конце сосредоточенной силой, нормальные напряжения изгиба для цельной трубы определяются по формуле [3]:

$$
\sigma_{u 0}^{\max }=\frac{M_{u 0}^{\max }}{I_{x_{0}}} \cdot y_{\max }=\frac{F \cdot l}{\pi \cdot R^{2} \cdot t}
$$

Для ослабленной трубы формула для определения нормальных напряжений в наиболее опасном сечении принимает следующий вид (см. рис. 9 и 10):

$$
\sigma_{u}^{\max }=\frac{M_{u}^{\max }}{I_{x}} \cdot\left|y_{1}\right|=\frac{2 \cdot F \cdot(z+c)}{\alpha \cdot R^{2} \cdot t} \cdot \frac{k_{3}}{k_{1}}
$$

Отношение выражений (19) и (20) позволяет установить степень уменьшения прочности изогнутой трубы при ее ослаблении, зависящую от геометрии и локализации выреза:

$$
\frac{\sigma_{u}^{\max }}{\sigma_{u 0}^{\max }=}=\frac{1}{1-\frac{\beta}{2 \pi}} \cdot \frac{k_{3}}{k_{1}} \cdot \frac{z+c}{l}
$$

\section{УСТОЙЧИВОСТЬ ЗАМКНУТЫХ ЦИЛИНДРИЧЕСКИХ ОБОЛОЧЕК}

Процесс радиального обжатия тонкостенной трубки с проволочной паковкой сопровождается изменением формы сечения трубки. В связи с этим необходимо рассмотреть вопрос потери устойчивости цилиндрических оболочек.

Выделим элемент срединной поверхности оболочки (рис. 11). Линии $\mathrm{A}_{1} \mathrm{CB}_{1}$ и $\mathrm{A}_{2} \mathrm{CB}_{2}$ представляют собой линии пересечения срединной поверхности с двумя взаимно перпендикулярными плоскостями, нормальными к поверхности и проходящими через нормаль к ней. Радиусы $\mathrm{R}_{1}$ и $\mathrm{R}_{2}$ являются радиусами кривизны линий $\mathrm{A}_{1} \mathrm{CB}_{1}$ и $\mathrm{A}_{2} \mathrm{CB}_{2}$. Величины, обратные радиусам, являются кривизнами оболочки: $\mathrm{K}_{1}=1 / \mathrm{R}_{1} ; \mathrm{K}_{2}$ $=1 / R_{2}$.

Из теории упругости известно [6], что на срединной поверхности оболочки всегда найдутся две взаимно перпендикулярные линии, кривизны которых обладают свойством экстремальности. Одна из них максимальна (обозначается индексом 1), а другая минимальна (обозначается индексом 2). Произведение этих кривизн, называемых главными, представляет собой гауссовскую кривизну, которой и описывается геометрия оболочки: $\mathrm{G}=\mathrm{K}_{1} \cdot \mathrm{K}_{2}$. Для замкнутых круговых цилиндрических оболочек типа труб, у которых $\mathrm{R}_{1}=\infty$ и $\mathrm{R}_{2}=\mathrm{R}=$ const, главные кривизны имеют следующие значения: $\mathrm{K}_{1}=0$; $\mathrm{K}_{2}=\mathrm{K} ; \mathrm{G}=0$. Положение произвольной точки на поверхности такой оболочки определяется двумя координатами: линейной $z$ вдоль образующей оболочки и угловой $\theta$ вдоль дуги $s$ постоянным радиусом R.

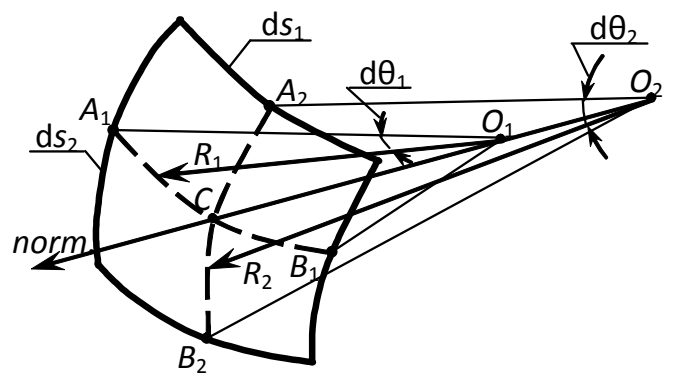

Рис. 11 Расчетная схема элементарного фрагмента срединной поверхности оболочки

В зависимости от отношения толщины оболочки $\mathrm{t} k$ радиусу кривизны срединной поверхности $\mathrm{R}$ оболочки делят на два вида: 1) толстые, когда $t / R \geq 1 / 30 ; 2$ ) тонкие, когда $1 / 30>t / R \geq 1 / 1000$. Толстые оболочки рассчитываются как упругое трехмерное тело. Тонкие оболочки, имеющие прогибы более четверти своей толщины, называются гибкими.

В инженерной практике расчет тонких оболочек произвольной формы производится двумя методами в зависимости от вида напряженного состояния: 1) по безмоментной теории; 2) по моментной теории.

Первый метод применяется при напряженном состоянии, которое характеризуется постоянством напряжений по толщине оболочки. В этом случае отсутствует изгиб оболочки, и в ней возникают лишь нормальные $\mathrm{N}_{1}$ и $\mathrm{N}_{2}$ и сдвигающие $\mathrm{S}_{1}$ и $\mathrm{S}_{2}$ силы (последние действуют по касательной к срединной поверхности).

Второй метод применяется при напряженном состоянии, когда в оболочке возникают изгибающие $\mathrm{M}_{1}$ и $\mathrm{M}_{2}$ и крутящие $\mathrm{T}_{1}$ и $\mathrm{T}_{2}$ моменты и поперечные силы $\mathrm{Q}_{1}$ и $\mathrm{Q}_{2}$ (последние действуют перпендикулярно к срединной поверхности).

На практике по безмоментной теории рассчитываются тонкостенные сосуды, резервуары с применением известного из курса сопротивления материалов уравнения Лапласа [3]. Этот метод значительно проще, чем расчет по полной, моментной теории, но он не всегда является достаточным и точным. Более точный расчет получают методом моментной теории. Обе эти теории являются линейными, и в теории упругости подробно разработаны методы расчета оболочек с применением обеих теорий [6].

В [3] рассмотрена задача об устойчивости кольца, сжатого радиальной равномерно распределенной нагрузкой, при которой круговая форма кольца становится неустойчивой, кольцо изгибается и, как считает автор, принимает примерно эллиптическую 
форму (рис. 12, а). Полагается, что местный радиус кривизны мало отличается от начального радиуса: $\rho \approx \mathrm{R}$. Результат, полученный для кольца, распространен на случай длинной трубы.

Как показывают эксперименты, для гибких замкнутых цилиндрических оболочек при их внешнем радиальном обжатии потеря устойчивости сопровождается образованием волнистой боковой поверхности оболочки (рис. 12, б). При этом возникают большие деформации, сопровождающиеся появлением не только напряжений изгиба, но и дополнительных напряжений, называемых цепными.

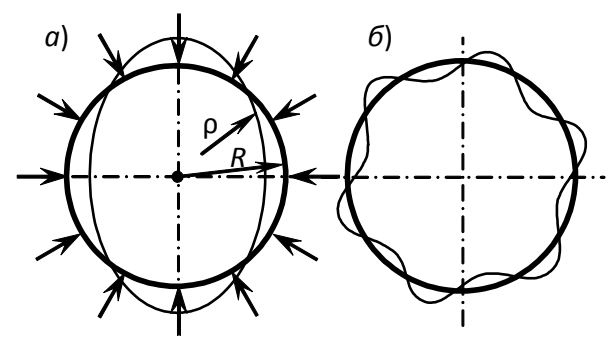

Рис. 12 Изменение формы кольца согласно линейной теории (а) и фактическое (б)

Существующие теории расчета оболочек, безмоментная и моментная, применимы лишь при малых деформациях, и дают представление только об устойчивости в малом. Поэтому полное решение такой задачи возможно лишь с применением нелинейной теории оболочек.

Технология изготовления материалов, предполагаемых к использованию в термоядерных реакторах [1], основана на упруго-пластической деформации тонкостенных длинномерных труб пошаговым радиальным обжатием. Для создания такой технологии необходима разработка теории расчета замкнутых цилиндрических оболочек бесконечной длины (тонкостенных труб пустотелых и с проволочной паковкой) на прочность и устойчивость в упругопластической области нагружения.

При помощи программы ANSYS смоделирован процесс деформирования цилиндрической оболочки с проволочной паковкой [7]. Потеря устойчивости такой оболочки сопровождается образованием волнистой боковой поверхности (рис. 13).

Подобные конструкции изучены не в полной мере и теоретически на данный момент не обоснованы.

В настоящее время авторами проводятся исследования напряженно-деформированного состояния цилиндрической оболочки и проволочной паковки при упруго-пластическом обжатии с применением нелинейной теории.

\section{УПРУГОЕ ВЗАИМОДЕЙСТВИЕ ПРОВОЛОК В ПАКОВКЕ}

В процессе радиального обжатия паковки взаимодействие параллельно уложенных проволок представим как взаимодействие цилиндров диаметром d, между которыми образуется контактная площадка в форме бесконечной прямоугольной полосы шириной $2 b_{k}$ (рис. 14) [8]. В качестве внешней нагрузки будем рассматривать распределенную нагрузку q, действующую вдоль образующей цилиндров.
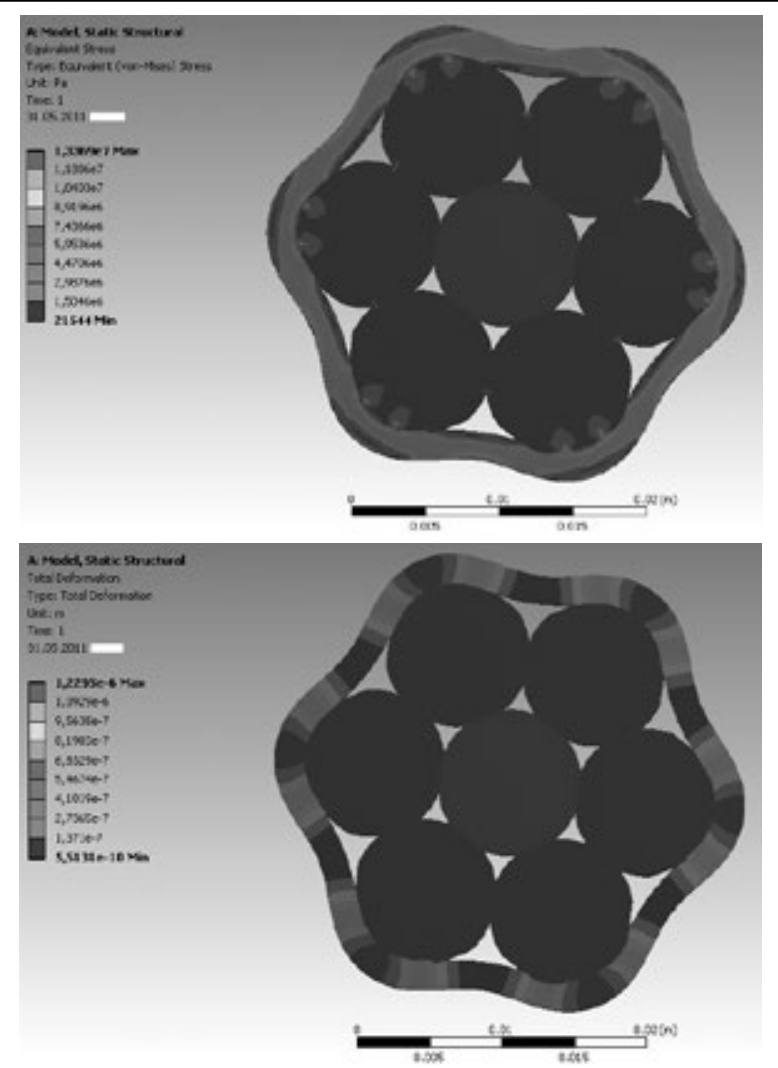

Рис. 13 Моделирование напряженного и деформированного состояния чилиндрической оболочки $c$ проволочной паковкой

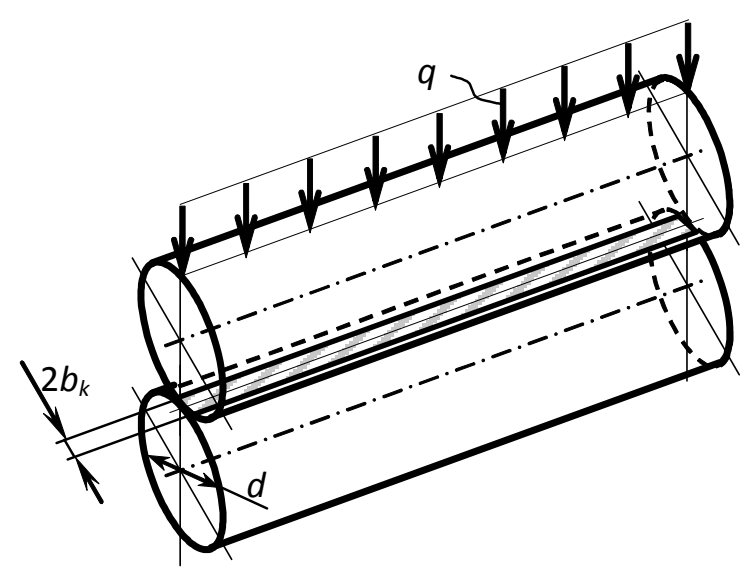

Рис. 14 Взаимодействие параллельных цилиндров

Давление $\mathrm{p}$ по ширине площадки контакта распределяется по закону полуэллипса (рис. 15) [8]:

$$
\left(\frac{p(x)}{p_{\max }}\right)^{2}+\frac{x^{2}}{b_{k}^{2}}=1
$$

Отсюда находим давление, действующее на расстоянии х от середины площадки контакта:

$$
p(x)=p_{\max } \cdot \sqrt{1-\frac{x^{2}}{b_{k}^{2}}}
$$




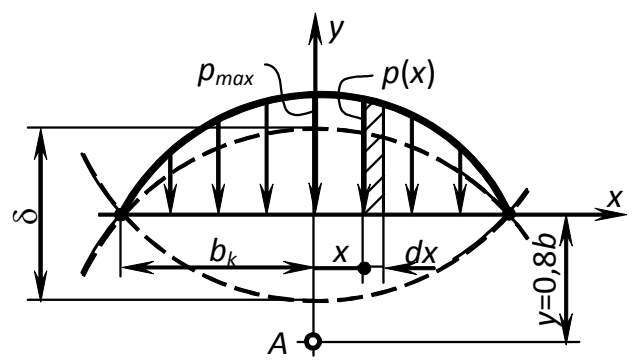

Рис. 15 Распределение давления по ширине площадки контакта

Выражение для распределенной нагрузки в интегральной форме имеет вид [8]:

$$
q=\int_{-b_{k}}^{b_{k}} p(x) \cdot d x
$$

Тогда с учетом равенства (23) после интегрирования в указанных пределах получим:

$$
q=\frac{p_{\max }}{b_{k}} \cdot \int_{-b_{k}}^{b_{k}} \sqrt{b_{k}^{2}-x^{2}} \cdot d x=\frac{\pi \cdot b_{k}}{2} \cdot p_{\max }
$$

Отсюда находим наибольшее давление, действующее в центре эллиптической площадки контакта:

$$
p_{\max }=\frac{2 \cdot q}{\pi \cdot b_{k}}
$$

Половина ширины площадки контакта равна [8]:

$$
b_{k}=0,8 \cdot \sqrt{q \cdot d \cdot \frac{1-\mu^{2}}{E}}
$$

где:

$\mu$ и Е - соответственно коэффициент Пуассона и модуль Юнга материала цилиндров (проволоки).
Тогда выражение (26) принимает вид:

$$
p_{\max }=0,8 \cdot \sqrt{\frac{q}{d} \cdot \frac{E}{1-\mu^{2}}}
$$

Формула для определения сближения цилиндров имеет следующий вид [8]:

$$
\delta=0,636 \cdot \frac{q}{E} \cdot\left(\ln \frac{d^{2}}{b_{k}^{2}}+0,814\right)
$$

С точки зрения напряжений при эллипсоидальном распределении давлений по ширине площадки контакта наибольший интерес представляет напряженное состояние в точках, расположенных на оси у. В этих точках все касательные напряжения на площадках, параллельных координатным осям, обращаются в ноль (в силу симметрии напряженного состояния относительно оси у). Поэтому нормальные напряжения на этих площадках являются главными, и при $\mathrm{y}=0$ в центре эллиптической площадки контакта формулы для их определения имеют следующий вид [9]:

$$
\begin{aligned}
& \sigma_{z}=-2 \mu \cdot p_{\max }=\sigma_{1} \\
& \sigma_{x}=-p_{\max }=\sigma_{2} \\
& \sigma_{y}=-p_{\max }=\sigma_{3} .
\end{aligned}
$$

Для площадки контакта в виде полосы такой точкой является точка $\mathrm{A}$ на рис. 15, лежащая на оси у и имеющая координату $\mathrm{y}=0,8 \mathrm{~b}$. В этой точке $\sigma_{\mathrm{ekw}}=0,6_{\mathrm{pmax}}$.

При помощи программы ANSYS смоделирован процесс упругого деформирования двух параллельно расположенных цилиндров и получена картина изменения напряжений в их контактной зоне (рис. 16).

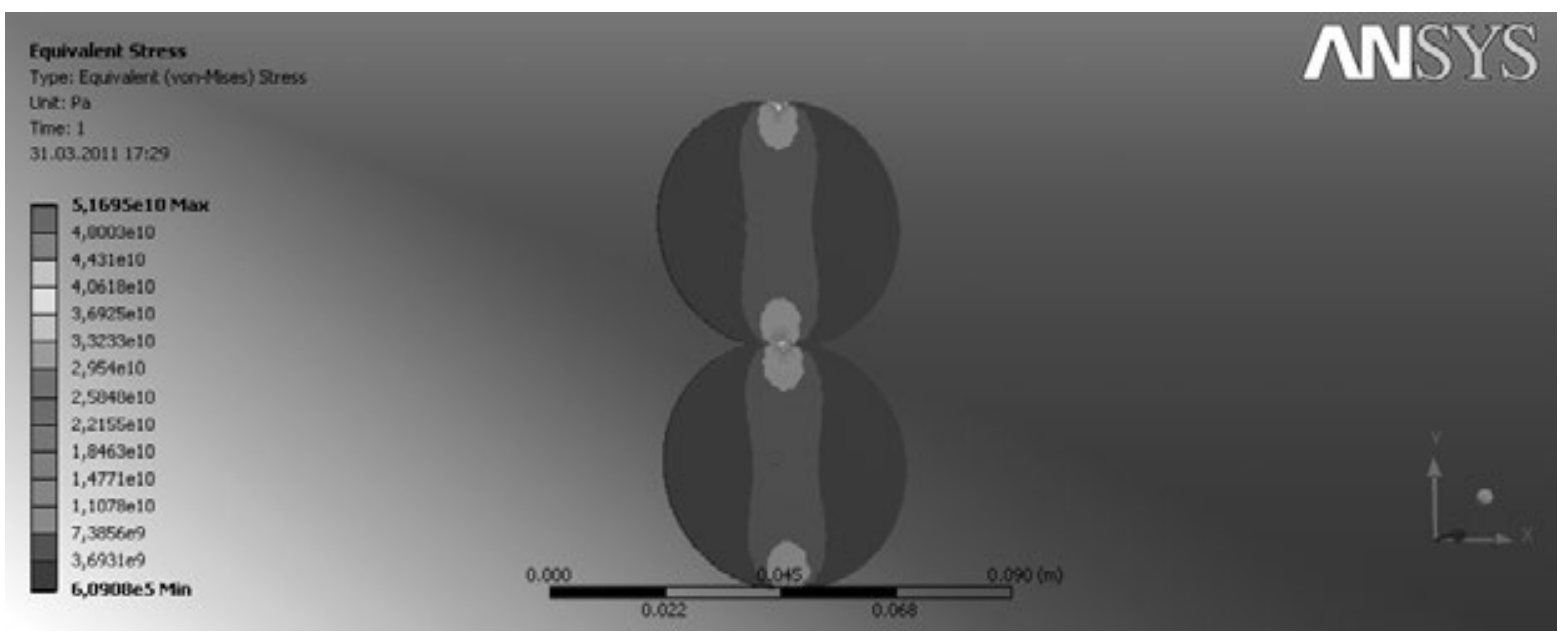

Рис. 16 Напряженное состояние цилиндров в контактной зоне 


\section{ЗАКЛЮЧЕНИЕ}

Технологию изготовления контактных соединений сверхпроводящих кабелей в металлической оболочке можно реализовать путем пошагового радиального обжатия медной трубки на пакет проводников.

Наиболее компактная паковка проводников формируется при упорядоченной укладке проволоки с использованием оболочки шестигранного профиля, однако следует принять во внимание, что в качестве трубных элементов конструкций наиболее часто используют круглый профиль.

Сравнительный расчет прочности по двум типичным схемам нагружения (сжатию и изгибу) цельной трубы и трубы ослабленной секционным вырезом позволил установить, что в обоих случаях увеличение угла разделки приводит к стремительному увеличению напряжений (снижению прочности) в ослабленном сечении, но место разделки при сжатии значения не имеет.

Смоделирован механизм радиального обжатия тонкостенной трубки с проволочной паковкой и обоснована необходимость использования нелинейной теории расчета тонкой цилиндрической оболочки на устойчивость.

Обжатие паковки исследовано на модели упругого взаимодействия параллельно расположенных цилиндров, между которыми образуются контактные площадки в форме прямоугольной полосы, и получены формулы для определения размеров контактной площадки, сближения цилиндров и максимального давления в контакте.

\section{ЛИТЕРАТУРА}

[1] O.P. Reut i nn. "Izostaticzieskoje obzhatie kontaktnych sojedinienij (KS) swierchprowodiaszczego kabiela magnitnych sistiem termojadiernogo rieaktora ITER", Int. Symp. IPM „Inzhynieria powierchnosti, nowyje poroszkowyje kompozicionnyje matierialy, swarka", Minsk, BY, 2009, pp. 131-134, cz. 2.

[2] A.Cz. Jakubowski i Cz.A. Jakubowski „Optimizacja pakowki mnogozhylnych kabielej w mietalliczeskoj oboloczkie" w Maszynostrojenie (Belarus), 2014, wyp. 28, pp. 137-140.

[3] W.I. Fieodosjew. Soprotiwlenie matierialow. Moskwa, RUS: MGTU, 1999, 591 p.

[4] A.Cz. Jakubowski i inn. "Rascziot gieomietriczeskich charaktieristik popieriecznych sieczienij oslablennoj truby" w Tieoreticzeskaja i prikladnaja miechanika (Belarus), 2011, wyp. 26, pp. 338-341.

[5] A.Cz. Jakubowski i Cz.A. Jakubowski. "Srawnitielnyj rascziot procznosti celnoj i oslablennoj truby", V Belarus Congr. TPM „Miechanika-2011”, Minsk, BY, 2011, pp. 477-480, t. 2.

[6] W.I. Samul. Osnowy tieorii uprugosti i plastycznosti. Moskwa, ZSRR: Wysszaja szkola, 1982, 264 p.

[7] A.Cz. Jakubowski i Cz.A. Jakubowski. „K woprosu rascziota zamknutych cylindriczeskich oboloczek na ustojcziwost" w Tieoreticzeskaja i prikladnaja miechanika (Belarus), 2012, wyp. 27, pp. 323-325.

[8] E.E. Pietiuszyk i inn. „Napriazhonnodieformirowannoje sostojanije $w$ jedinicznom kontakcie pri uprugom dieformirowanii pronicajemych matierialow na osnowie prowoloki w widie tiel namotki", Wiesti Akad. Nauk Belarusi, ser. Fiz.-Techn. Nauk, № 2, pp. 10-15, 2002.

[9] S.D. Ponomariow. Rasczioty na procznost w maszynostrojenii. Moskwa, ZSRR: Maszgiz, tom 2, 1958, 974 p.

\section{dr inż. Andrzej Jakubowski}

Maritime University of Szczecin, Department of Technical Mechanics IPNT WM

Wały Chrobrego 1-2, 70-500 Szczecin, POLAND

e-mail: a.jakubowski@am.szczecin.pl 